\title{
La créativité peut-elle devenir un paradigme pédagogique émancipateur ?
}

Sébastien Charbonnier, Université Lille, EA 4354 - Centre Interuniversitaire de Recherche en Education de Lille (CIREL), France

L'éloge de la créativité se fait de plus en plus unanime : elle est désignée comme une capacité désirable qu'il serait bon de former chez les individus, et ce dès la formation scolaire. D’un point de vue épistémologique, on ne peut que se réjouir de ce « tournant » pédagogique, dès lors que l'on considère que l'appropriation réelle des savoirs suppose leur construction par les élèves eux-mêmes. En mettant ainsi l'accent sur la créativité, la pédagogie peut mettre au centre les puissances créatives de l'individu, puissances aussi indispensables à la transmission de savoirs anciens qu'à la compétence à produire des savoirs nouveaux.

Mais, dans les questions éducatives, la naïveté n'est pas de mise : il serait terrible de passer à côté des impensés politiques de cette valorisation nouvelle de la créativité. Si Adobe ou Apple lancent de grandes enquêtes sur la créativité, c'est aussi parce que cette compétence accueille bien des fantasmes dans l'imaginaire actuel de l'économie. Au sein de la nouvelle économie de la connaissance, la créativité serait la nouvelle ressource (psychique) dans un monde où les ressources matérielles se font de plus en plus rares. De fait, le fort potentiel de plus-value de l'idée « géniale » fonctionne suivant le mythe de l'Eldorado : une seule bonne idée, et c'est la fortune...

Il faut donc être très vigilant au risque d'une contradiction profonde : l'injonction à la créativité est extrêmement anxiogène, car l'ordre «Soyez créatifs ! » ne repose sur aucune procédure à suivre (même si l'on essaie d'en formaliser). En effet, dans l'apprentissage de conduites, l'absence de règles procédurales entraîne ipso facto le verbalisme, comme le souligne Isräl Scheffler : on dit ce qu'il faut faire sans jamais apprendre comment le faire (116-120). C'est une situation de souffrance psychique réelle qu'il n'est pas désirable de faire subir à des individus.

Par contraste, avec ces dangers d'aliénation nouvelle, cet article voudrait interroger, avec les armes de la philosophie critique, comment la créativité peut devenir un paradigme émancipateur dans l'éducation et l'instruction. On verra que, prise au sérieux d'un point de vue éthique, la créativité est une compétence qui permet non seulement d'être un acteur de la connaissance en train de se faire, mais aussi un citoyen critique vis-à-vis des aliénations nouvelles de l'économie de la connaissance. 
Je souhaiterais donc procéder à une analyse conceptuelle de la créativité en trois moments qui sont autant de dimensions du concept : (1) la créativité comme construction ontologique (la pensée est ajout de quelque chose dans le monde : si je pense réellement, je ne suis plus le même qu'avant donc j'ai transformé le monde, à commencer par moi-même - qui fais partie du monde), (2) la créativité comme nouveauté épistémique (penser c'est toujours penser autrement qu'on ne pensait avant) et (3) la créativité comme imagination éthique (la puissance d'imaginer est fondamentale pour évaluer ce qu'est une vie bonne ; en ce sens, créer c'est cultiver la force de l'imaginaire, indispensable pour toute éducation libérale - on le sait depuis La Boétie : la servitude provient d'abord d'une carence de l'imaginaire).

En prenant pour exemple précis la didactique de la philosophie, je voudrais montrer comment s'articulent ces différentes dimensions de la créativité entendue comme disposition émancipatrice. Ainsi, la caractérisation de l'activité philosophique comme «P-creativity», pour reprendre le terme de Boden, pourra prendre un sens très précis et exigeant : il ne saurait y avoir d'apprentissage réel s'il n'y a pas transformation véritable du sujet pensant ${ }^{1}$.

\section{Penser, c'est se créer un soi (dimension ontologique)}

Ce premier moment sur la dimension ontologique de la créativité s'oppose au premier grand modèle spiritualiste et statique de la pensée : penser ce serait contempler le vrai (Aristote). Il faut opposer à cette croyance au «mythe du donné » (Bachelard 10) une conception constructiviste des objets du savoir et des sujets savants.

\subsection{Se mettre en mouvement}

Comment met-on les élèves en activité créatrice ? De manière très concrète, la question des effectifs est le premier élément qui se dégage massivement des observations faites dans les classes de Terminale en France : on ne crée pas à trente-cinq ensemble. Il apparaît nettement que la surcharge des classes induit la magistralité pédagogique. Si l'on veut mettre les élèves en dynamique de créativité, il faut travailler par petits groupes.

Par exemple, les expériences de participation au Festival philosophique du film (Eidolon), qui comprend une partie exclusivement réservée aux lycéens, montrent la richesse du travail en groupe et sur le long terme dans une entreprise de créativité. De ce point de vue, on peut esquisser l'hypothèse suivante : former l'imagination par la création artistique (ici, un court-métrage cinématographique) est utile pour apprendre à philosopher, voire est déjà philosopher. En créant un objet symbolique, les élèves font l'expérience d'une création de soi. Or, cela prend du temps : les élèves ont plusieurs mois pour tâtonner (l'errance est cruciale pour créer véritablement), se corriger (le pronominal est d'essence !), filer plusieurs pistes, etc. 
Apprendre la créativité n'est ainsi possible que si sont dégagées des vacuoles temporelles où peuvent se déployer le tâtonnement, l'erreur, la réorientation des idées, etc. Cette nécessité du ralentissement des rythmes va à l'encontre de la forme scolaire, trop souvent empressée et compétitive.

La créativité a donc des propriétés objectives de résistance à l'aliénation moderne suprême : l'accélération de nos modes de vie (Rosa). En ce sens, la créativité forme l'esprit critique réellement, car elle dispose bien l'individu dans son esprit et son corps en formant en lui des aptitudes qui l'aident à résister à un certain régime de vitesse.

\subsection{Comment évaluer ce qui a eu lieu?}

La créativité pose de manière très aiguë la question de l'évaluation. Dès lors que l'idée de nouveauté est au cœur de la créativité, comment évaluer - si l'on considère qu'une évaluation se fait toujours en référence à un système de valeurs donné ? On voit bien le paradoxe : un acte créatif déborde les cadres préexistants qui sont pourtant nécessaires à toute évaluation.

Dans cette première dimension ontologique, le critère sera alors celui des sensations. Pour évaluer la créativité, il est fort utile de recourir à un concept de John Dewey : la valuation. De fait, la valeur de ce que dit ou produit un individu ne doit pas être jugée au regard d'une norme extrinsèque (évaluation), il faut plutôt considérer la valeur énergétique de ce que cela nous fait de manière immanente (valuation). Par exemple, répéter une idée rationnelle « vraie » par cœur sans la comprendre peut être évalué positivement dans un système qui valorise la bonne réponse, mais cela n'a aucune valeur formative pour le psychisme de l'élève.

Cette distinction entre «évaluation » se référant à un système de normes extérieur à la situation, et «valuation » exprimant le fonctionnement intrinsèque d'un dispositif invite donc les individus à devenir capables d'une forme de connaissance de soi-même. On pourra ici distinguer les sensations internes et celles externes. D'un côté, valuer la créativité suppose de se demander si je sens que je suis en train de créer, si je fais l'expérience de la «satisfaction intérieure » (Spinoza 317) : suis-je joyeux parce que je suis en train d'exercer mes forces ? De l'autre côté, les sensations externes sont des indices aussi très précieux : je perçois que j'ai créé quelque chose (poïesis). La médiation de l'objet comme création vaut objectivation de mes forces : elle est donc une condition nécessaire à la subjectivation. L'objet est bien une médiation indispensable à l'œuvre de soi. 


\section{Penser, c'est penser autrement (dimension épistémologique)}

Ce deuxième moment sur la dimension épistémologique de la créativité s'oppose au second grand modèle spiritualiste de la pensée : penser ce serait viser le vrai (Descartes) ou obtenir un consensus (Habermas). Il faut opposer à cet amour des solutions une philosophie du problème qui met l'accent sur les écarts et les surprises dans l'ordre de la pensée.

\subsection{Brasser pour pouvoir créer}

Il faut souligner ici le rôle crucial du paradoxe : la pensée créative n'a jamais lieu dans les chemins déjà tracés, elle suppose un pas de côté. Qu'est-ce qui peut me faire faire ce pas de côté qui m’obligera à parcourir d'autres étendues ?

Tout individu souhaitant créer doit partir à la quête d'un point émotionnel réellement présent à partir duquel il pourra construire. Ce point, personne ne le connaît a priori. Il n'y a pas de méthode - au sens cartésien d'un chemin qui assure le succès. Seul le fait de brasser de tout peut permettre l'émergence du nouveau. Gottfried Wilhelm Leibniz l'a dit sans ambages : le faux a une positivité, il faut même dire que plus il y aura de faux, plus on aura de chances de trouver du vrai. C'est ce que signifie l'éloge de la culture dans les Nouveaux essais : « qui aura lu plus de romans ingénieux, entendu plus de narrations curieuses, celui-là dis-je, aura plus de connaissance qu'un autre, quand il n'y aurait pas un mot de vérité en tout ce qu'on lui a dépeint ou raconté ; car l'usage qu'il a de se représenter dans l'esprit beaucoup de conceptions ou idées expresses et actuelles le rend plus propre à concevoir ce qu'on lui propose » $(\mathrm{IV}, 1)$. De manière combinatoire, Leibniz insiste sur les puissances du faux comme mécanisme producteur de nouvelles idées. Leur fécondité pour la pensée tient au fait que l'esprit a besoin de matériaux, quels qu'ils soient, pour opérer par combinaisons, transports et rapprochements.

En bref, la culture d'un domaine est fondamentale pour la créativité dans ce domaine. Le concept de «création » chez Leibniz permet de lutter contre l'idéologie néoromantique de la virginité créatrice (le mythe de l'ignorance féconde, le refus des influences, etc.). C'est ici qu'il s'oppose le plus vigoureusement à Descartes. Ces deux philosophes rationalistes considèrent des mélanges de vrai et de faux, mais en les articulant différemment. (a) Pour Descartes, dès lors que le moindre faux diffus dans un ensemble vrai est suspecté, c'est tout l'ensemble vrai qui sera rejeté : méthode du doute hyperbolique. Ce qui compte, c'est la conformité au vrai. (b) Pour Leibniz, au contraire, dès lors qu'il y aura le moindre vrai diffus dans un ensemble faux, c'est tout l'ensemble faux qui sera accepté. Le perspectivisme leibnizien comporte une conséquence décisive pour les visées des apprentissages : il est indispensable de supprimer l'allégeance à la vérité comme telle : c'est bien l'importance que l'on donne à ses idées qui constitue la forme du faux - ou la sottise - et s'oppose aux puissances 
du faux. Ce qui compte, c'est le fait qu'il y ait des idées.

Or, le premier objectif en termes de créativité, c'est d'avoir (juste) une autre idée avant de viser une idée juste. De plus, le faux possède une faculté de déstabilisation : par son brassage permanent, il protège de l'allégeance sclérosante au vrai. L'expérience joyeuse fournit un matériau qui est la condition de possibilité d'entrer dans une aventure d'idées qui permet de refournir des données, recharger le monde en données, faire circuler quelque chose, autant que possible et si peu que ce soit. Sans cela, point de créativité, point de pensée.

\subsection{Qui peut évaluer ce qui est créé?}

De nouveau, prendre au sérieux la créativité, dans sa dimension épistémologique cette fois-ci, permet de repenser pédagogiquement l'évaluation. En effet, le problème de la créativité met au cœur des apprentissages la question de l'horizon d'attente. Il devient nécessaire de se demander: quel public vise la création des élèves ? Cette attention au destinataire des productions est précieuse, car c'est le sens même d'une action qui se joue ici. En effet, produire en imaginant un destinataire confère du sens à mon geste : cela va à l'encontre de l'artificialité des productions scolaires destinées uniquement à être lues et notées par un professeur. Charlotte Nordmann dit abruptement : comment peut-on aimer écrire dans le seul but de recevoir une note ? Cette absurdité de certaines productions scolaires peut être problématisée lorsqu'un souci pour la créativité est réintégré au cœur des apprentissages. Par contraste, le système scolaire repose actuellement sur des modes de production qui suscitent le dégoût chez les destinataires : le vrai problème des productions scolaires, c'est que les professeurs n'aiment pas les lire! Cela est particulièrement vrai avec les productions scolaires en philosophie : il y a un malaise profond de la correction chez les enseignants de philosophie (Rayou).

De plus, la créativité est la condition d'un désir d'apprendre chez l'autre. Elle est une condition fondamentale du désir d'apprendre entre enseignants et apprenants : en effet, la créativité des élèves crée chez l'enseignant le désir d'apprendre de ses élèves (et non plus seulement aux élèves). Cette réciprocité est absolument fondamentale dans un processus d'apprentissage (Charbonnier, Érotisme, 135-146). Nouveau critère de valuation intrinsèque : l'intérêt d'autrui est une sanction immanente de la créativité, c'est un signe objectif qui ne trompe pas.

L'horizon de formation de l'esprit critique est ici encore très prometteur. C'est l'éthos démocratique qui se met en place avec la créativité : les élèves peuvent apprendre à apprendre à d'autres, ils ne sont plus acculés à la posture passive de receveurs du savoir. Pour un enseignant, le premier bonheur d'avoir des élèves créatifs est la perspective qu'il va pouvoir apprendre des choses d'eux. Il faut prendre au sérieux cette exigence et se demander quels 
savoir-être disposent à l'exercice de la vigilance politique citoyenne, et quels savoir-être disposent au contraire à la passivité, à l'acceptation résignée de jugements d'experts qui «savent forcément mieux que nous »? Je soutiens que le souci pour la créativité induit des modalités épistémologiques de circulation du savoir résolument démocratiques, car elles favorisent l'égalité épistémique entre les individus - nous avons des choses à nous apprendre les uns des autres - contre toute forme de despotisme intellectuel - je sais mieux que toi et il n'y a que moi qui puisse t'apprendre (Rancière).

\section{Penser, c'est faire fonctionner, brasser des objets symboliques (dimension éthique)}

Ce dernier moment sur la dimension éthique de la créativité s'oppose au troisième grand modèle spiritualiste de la pensée : penser ce serait juger (Kant). Il faut opposer à cet amour du jugement une pensée productrice qui fait exister ce qui n'existait pas encore.

\subsection{Gagner en liberté d'usage de la culture}

Ultimement, c'est bien une perspective éthique qui se dessine avec la mise en avant de la créativité dans les apprentissages. Il s'agit d' « en finir avec le jugement » (Deleuze 168). Le modèle du tribunal pour concevoir ce que signifie penser ne fait rien advenir, il ne légifère que sur du déjà existant. «Le jugement empêche tout nouveau mode d'existence d'arriver. Car celui-ci se crée par ses propres forces, c'est-à-dire les forces qu'il sait capter, et vaut par luimême, pour autant qu'il fait exister la nouvelle combinaison. C'est peut-être là le secret : faire exister, non pas juger » (Deleuze 168). Au système du jugement s'oppose la perspective critique de la créativité. Par critique, il faut entendre une capacité à discriminer les matériaux utiles pour une création nouvelle. C'est donc la liberté d'un certain usage de la culture qui est ici en jeu, contre l'effet délétère d'un rapport révérencieux à la « grande » culture. Supprimer l'allégeance à la culture légitime : cela ne signifie pas l'ignorer (on l'a vu précédemment), mais savoir la respecter - qui ne veut pas dire la révérer (Charbonnier, Philosophie).

La culture fait sens dans la mesure où elle se met à vivre en moi : je suis transformé par elle si et si seulement je la digère. Alors je pourrai produire quelque chose. Mais il y a un corolaire capital à cela : la culture elle-même se transforme nécessairement, elle ne demeure pas intacte dans ce contact vivifiant avec les individus concrets. Se former auprès des œuvres culturelles signifie ici innutrition donc transformation. La culture devient aliment, et non objet précieux à conserver; elle n'est plus objet de musée à contempler béatement, mais nourriture jubilatoire à croquer, lécher, sucer, avaler, mâcher. Bref, elle n'est plus morte elle est vivante. La créativité repose sur un tel rapport nourricier à la culture - qui devient aliment pour créer demain et non objet sacré d'hier. 
Mais renoncer au jugement, est-ce à dire que tout se vaut ? Il faut prendre très au sérieux ce risque qui pourrait poindre : l'éloge de la créativité pour elle-même induit-il le relativisme? Gilles Deleuze renverse l'accusation : «N'est-ce pas plutôt le jugement qui suppose des critères préexistants (valeurs supérieures), de telle manière qu'il ne peut appréhender ce qu'il y a de nouveau dans un existant, ni même pressentir la création d'un mode d'existence ?» (168). Sans doute conscient de cette impuissance, le jugement produit activement les conditions de son efficacité : il empêche tout nouveau mode d'existence d'arriver - évitant ainsi de se retrouver confronté à ce qu'il ne peut penser. «Faire exister, non pas juger », crie Deleuze qui explique : «S'il est si dégoûtant de juger, ce n'est pas parce que tout se vaut, mais au contraire parce que tout ce qui vaut ne peut se faire et se distinguer qu'en défiant le jugement » (169). La critique est un acte de créativité au sens plein : elle apporte au monde et donne à voir les valeurs qu'elle défend en les agissant. Nouvelle illustration de la pertinence de la distinction de Dewey mettant en avant la valuation.

\subsection{Un exemple de situation didactique contre-intuitive}

Pour terminer cet article, je souhaiterais partager un dispositif d'apprentissage de l'écriture. Il s'agit d'une situation didactique éprouvée dans plusieurs classes de philosophie.

Apprendre à écrire philosophiquement est souvent un impensé en didactique de la philosophie. La pratique de l'écriture se heurte de plus à un phénomène massif de marchandisation des «corrigés » sur le net, ce qui rend problématiques les devoirs faits à la maison (DM). Dès lors, au lieu de s'horrifier du fait que les élèves peuvent aller voir sur Internet, éventuellement copier, un exercice formateur consiste à les forcer au jeu de l'assemblage afin de leur faire prendre conscience du mécanisme imitatif et inventorial de la pensée - l'ars inveniendi se fonde sur l'ars inventoriendi, dirait Leibniz. (IV, 21) Contre le mythe selon lequel je suis l'auteur sui generis de mes idées, ce travail de «copier/coller» explicite permet de démythifier les puissances de la pensée : lorsque je crois avoir une idée «à moi », je ne fais que témoigner de mon inconscience du mécanisme complexe de combinaison et de réagencement qui s'est opéré à partir des rencontres que j'ai faites. C'est une certaine conception de la pensée créative qui est en jeu.

Dans un souci d'entrée progressive dans l'écriture réflexive philosophique, ce premier exercice consiste non pas à poser une question, mais à donner un concept ou un couple de concepts en invitant les élèves à rédiger un article succinct dessus - même partiel. Ainsi, les élèves sont invités à chercher sur Internet des éléments de définition et d'explication de la notion. La règle cruciale est qu'ils n'ont pas le droit d'écrire un seul mot qui soit d'eux. Les deux pages sont alors un agencement de «blocs » d'écriture, dont les élèves devront citer 
explicitement les sources. Cet exercice prend également acte des mutations de l'écriture (manuscrite $v s$ électronique), étudiées par Pierre Billouet dans L'éducation scripturale. De la plume au clavier.

On passe ainsi de l'écriture perçue comme « personnelle », où les tentatives de fraudes sont tentantes et interdites, à une écriture de «compilation». Cette priorité accordée à l'ingestion sur la production, fondée ontologiquement, a connu divers heurs pédagogiques jadis - on peut penser à la pratique du «pastiche » en cours de littérature. Quelles sont les vertus d'un tel exercice ? J'en relèverai au moins quatre. a) Contre l'idée que l'on pense par soi-même (ce «par » est très mystérieux), cet exercice problématise in vivo que, pour créer de la pensée, il faut lire, et surtout élire ce qui est pertinent et forme sens - d'où l'importance d'une contrainte forte sur la longueur du texte attendu. Lectio puis electio : on arrive à la définition d'intellectio. Contre un certain individualisme narcissique, cette expérience fait donc sentir aux élèves qu'on ne pense jamais seul. b) Le problème de l'écriture comme telle est reporté à plus tard. Il ne s'agit pas de refouler cette difficulté, mais l'exercice permet de ne pas perdre d'emblée certains élèves pour la seule raison qu'ils n'aiment pas écrire. c) Un tel travail permet une éducation à l'usage d'Internet. Comme tout outil technique puissant, Internet offre des perspectives de libération et des forces d'asservissement; à ce titre, l'exercice est un moment privilégié pour discuter avec les élèves de la manière dont ils s'y prennent et pour former à un usage critique du web. d) Enfin, ces travaux ouvrent la perspective d'un travail collectif qui peut déboucher sur une création à part entière. Par exemple, une classe peut se donner comme objectif de corriger et d'améliorer un article de l'encyclopédie participative Wikipedia. Une telle expérience est vécue comme inédite par les élèves dans leur rapport à l'autorité : ils travaillent à produire un texte qui fait sens pour eux, car il est utile pour d'autres. La finalité de leurs efforts n'est pas d'être lu (souvent avec dégoût et tristesse) par un professeur et de se voir attribuer une note, mais par des milliers de lecteurs anonymes et heureux d'apprendre.

\section{Conclusion}

La question radicale qu'il faut se poser est «pourquoi crée-t-on ? ». C'est le sens de l'institution scolaire qui est interrogé par le choix de mettre la créativité au cœur des apprentissages. L'horizon du concept de créativité est, in fine, politique : c'est pour former les citoyens d'une véritable démocratie qu'il faut s'intéresser à la créativité à l'école. En effet, s'il n'y a jamais pensé, l'individu ne peut conquérir la force d'inventer par lui-même donc reste dans un état de minorité (au sens kantien) : la politique est une activité pratique qui suppose 
invention puisqu'elle est décision de réaliser collectivement des imaginaires - ce n'est ni vrai ni faux puisque c'est un acte.

Dit autrement, s'il y a bien un domaine où la créativité est indispensable, c'est dans la vie politique démocratique : les formes du bien-être collectif sont toujours à penser entre citoyens. Rien ne nous est interdit: il n'y a que l'absence d'exemples antérieurs et notre manque d'imagination qui nous empêchent de construire des mondes meilleurs.

\section{Bibliographie}

Bachelard, Gaston. La formation de l'esprit scientifique. Paris : PUF, 1938.

Billouet, Pierre. L'éducation scripturale. De la plume au clavier. Paris : Harmattan, 2010.

Boden, Margaret. The Creative Mind. Myths and Mechanisms (2e éd.). New York:

Routledge, 2003.

Charbonnier, Sébastien. Que peut la philosophie ? Paris : Seuil, 2013.

---. L'érotisme des problèmes. Lyon : ENS, 2014.

Deleuze, Gillet. Critique et clinique. Paris : Minuit, 1993

--- et Félix Guattari. Qu'est-ce que la philosophie ? Paris : Minuit, 1991.

Dewey, John. The Theory of Valuation. Carbondale : Southern Illinois UP, 2008.

Leibniz, Gottfried Wilhelm. Nouveaux essais sur l'entendement. Paris : GF, 1966.

Nordmann, Charlotte. La fabrique de l'impuissance 2. L'école, entre domination et émancipation. Paris : Amsterdam, 2007.

Rancière, Jacques. Le maître ignorant. Paris : Fayard, 1987.

Rayou, Patrick. La «dissert' de philo». Sociologie d'une épreuve scolaire. Rennes : PUR, 2002.

Rosa, Hartmut. Accélération. Une critique sociale du temps. Paris : La Découverte, 2010.

Scheffler, Israël. Le langage de l'éducation. Tr. M. Le Du. Paris : Klincksieck. 2003 [1989].

Spinoza, Baruth. Éthique. Tr. B. Pautrat. Paris : Seuil, 1998 [1677].

\footnotetext{
NOTE

${ }^{1}$ Ce point fut souvent mal compris par les objecteurs de la définition de la philosophie comme « création de concepts » (Deleuze et Guattari) : ils croyaient que les auteurs associaient la pensée véritable à la seule $\mathrm{H}$ creativity - « vous ne prétendez pas faire des élèves de futurs Platon tout de même ».
} 